\title{
Finite Density QCD: a New Approach
}

\author{
Vicente Azcoiti \\ Departamento de Física Teórica, Universidad de Zaragoza, Cl. Pedro Cerbuna 12, \\ E-50009 Zaragoza (Spain) \\ E-mail: azcoiti@azcoiti.unizar.es
}

\section{Giuseppe Di Carlo}

INFN, Laboratori Nazionali del Gran Sasso, 67010 Assergi,(L'Aquila) (Italy)

E-mail: gdicarlo@lngs.infn.it

\author{
Angelo Galante \\ INFN, Laboratori Nazionali del Gran Sasso, 67010 Assergi,(L'Aquila) (Italy) and \\ Dipartimento di Fisica dell'Università di L'Aquila, 67100 L'Aquila (Italy) \\ E-mail: galante@lngs.infn.it \\ Víctor Laliena \\ Departamento de Física Teórica, Universidad de Zaragoza, Cl. Pedro Cerbuna 12, \\ E-50009 Zaragoza (Spain) \\ E-mail: Laliena@unizar.es
}

\begin{abstract}
We introduce a new approach to analyze the phase diagram of QCD at finite chemical potential and temperature, test it in the Gross-Neveu model at finite baryon density, and apply it to the study of the chemical potential-temperature phase diagram of QCD with four degenerate flavors of Kogut-Susskind type.
\end{abstract}

KEYWORDs: lat lgf ssb. 


\section{Contents}

1. Introduction 1

2. Theory scheme 2

2.1 The action 3

2.2 Symmetries of the partition function

2.3 The extension to imaginary y 6

2.4 Determination of the critical chemical potential near the continuum 8

2.5 Two other alternative ways for extracting the critical $\mu$ : the $(\beta, y)$ and $(\beta, x)$ planes

2.6 Vacuum expectation values on the phase transition line 11

3. The Gross-Neveu model at finite density 11

4. Four flavor QCD at finite $\mathrm{T}$ and $\mu$

4.1 The $(x, y)$ plane 17

4.2 The $(\beta, y)$ plane

5. Conclusions and comments 19

\section{Introduction}

Hadronic matter at high density and finite temperature is expected to undergo one or more phase transitions from a confined phase to a quark-gluon plasma phase, and eventually a color superconducting phase due to a (weak) attractive channel between quarks of different colors, that could lead to the formation of quark pairs, analogous of Cooper pairing in solid state systems at low temperature. The behavior of hadronic matter at high density is also relevant in astrophysics, for the study of neutron stars and extremely compact objects, and in cosmology, for the understanding of phase transitions in the early Universe. Furthermore the new states of matter should be partially accessible to direct experimental verification in the heavy ion collision experiments.

These features of finite density QCD can be studied only nonperturbatively. Unfortunately the lattice approach, the most powerful tool to perform first principles, nonperturbative studies, is affected in the case of finite density QCD by the well known sign problem, that has prevented for a long time any step toward its understanding. Indeed the moderate optimism generated by the finding of a first order phase transition at high density in the strong-coupling limit [近] using the Glasgow reweighting method, was actually ill-founded [2]. 
From the accumulated experience it was clear that the only way to minimize the effect of the phase of the fermion determinant in the integration measure of finite density QCD would be to work in the limit of large fermion masses. In fact taking the limit of fat QCD and using the MFA approach [3] in the numerical simulations, we were able to predict a tentative phase diagram for fat QCD [4] which qualitatively confirmed the theoretical expectations.

This was the rather unpleasant situation of three-color QCD at finite density until two years ago, when Philippe de Forcrand and Owe Philipsen [5], and later on D'Elia and Lombardo [6], reported some reliable results for light quarks, obtained from simulations at imaginary chemical potential where the sign problem, as well known, is absent. Fodor and Katz have also reported interesting results [7] obtained within a modified version in twoparameter space of the Glasgow re-weighting technique [1] which, due to the re-weighting procedure, can be applied only to relatively small lattice sizes. A modified version of the last approach, which makes use of a Taylor expansion in the chemical potential, has produced also interesting results for larger lattice sizes in the small $\mu / T$ region [8].

This paper is devoted to the presentation of a new approach to simulate QCD at finite temperature and baryon density, and to the application of this approach to the four-fermion model in three dimensions and to QCD with four degenerate quarks. Even if our theory scheme can resemble in some aspects the imaginary chemical potential approach, its range of applicability is at first sight much wider, as will become apparent after reading section 2 , which we devote to a detailed exposition of the approach. Section 3 contains the results of a test worked on the three-dimensional Gross-Neveu model at finite baryon density and in the large number of flavors limit. This model shows a non trivial phase diagram in the $T, \mu$ plane [9] and in addition, in the large $\mathrm{N}$ limit, can be analytically solved, thus allowing to confront results obtained within our approach with exact ones. In section 4 we show our results for the phase diagram of QCD at finite chemical potential with four degenerate flavors of Kogut-Susskind type. The data reported in this section were obtained from simulations at values of the gauge coupling and fermion masses in the scaling region. Last section is devoted to collect our main conclusions and to discuss possible issues for near future.

\section{Theory scheme}

The lattice action for QCD at finite chemical potential $\mu$ and staggered fermions is

$$
\begin{aligned}
S & =S_{\mathrm{P} G}+\frac{1}{2} \sum_{n} \sum_{i=1}^{3} \bar{\psi}_{n} \eta_{i}(n)\left(U_{n, i} \psi_{n+i}-U_{n-i, i}^{\dagger} \psi_{n-i}\right) \\
& +\frac{1}{2} \sum_{n} \bar{\psi}_{n} \eta_{0}(n)\left(e^{\mu} U_{n, 0} \psi_{n+0}-e^{-\mu} U_{n-0,0}^{\dagger} \psi_{n-0}\right)+m \sum_{n} \bar{\psi}_{n} \psi_{n}
\end{aligned}
$$

where $S_{\mathrm{P} G}$ is the standard Wilson action for the gluonic fields and $\eta_{\mu}(n)$ the Kogut-Susskind phases. The temporal part of the fermionic action can also be written as 


$$
\begin{aligned}
S_{\tau} & =\cosh (\mu) \frac{1}{2} \sum_{n} \bar{\psi}_{n} \eta_{0}(n)\left(U_{n, 0} \psi_{n+0}-U_{n-0,0}^{\dagger} \psi_{n-0}\right) \\
& +\sinh (\mu) \frac{1}{2} \sum_{n} \bar{\psi}_{n} \eta_{0}(n)\left(U_{n, 0} \psi_{n+0}+U_{n-0,0}^{\dagger} \psi_{n-0}\right)
\end{aligned}
$$

The contribution proportional to $\cosh (\mu)$ in $S_{\tau}$ is a fermionic bilinear defined through a matrix $i \Lambda_{\tau}$ with $\Lambda_{\tau}$ a hermitian matrix which anticommutes with $\gamma_{5}$. The contribution proportional to $\sinh (\mu)$ is also a fermionic bilinear defined through the hermitian matrix $\bar{\Lambda}_{\tau}$. Since the space-like part of the fermionic action is also a bilinear defined by the matrix $m I+i \Lambda_{s}$, with $\Lambda_{s}$ hermitian, it is evident that the introduction of a chemical potential $\mu$ drive us to a complex fermion effective action. At the same time it is also obvious that an imaginary chemical potential solves the sign problem since $\cosh (\mu)$ becomes the trigonometric cosine and $\sinh (\mu)$ the trigonometric sine, but the last picking up a factor of i.

\subsection{The action}

Let us now define the following generalized action

$$
S=S_{\mathrm{P} G}+\frac{1}{2} \sum_{n} \sum_{i=1}^{3} \bar{\psi}_{n} \eta_{i}(n)\left(U_{n, i} \psi_{n+i}-U_{n-i, i}^{\dagger} \psi_{n-i}\right)+S_{\tau}(x, y),
$$

with

$$
\begin{aligned}
S_{\tau}(x, y) & =x \frac{1}{2} \sum_{n} \bar{\psi}_{n} \eta_{0}(n)\left(U_{n, 0} \psi_{n+0}-U_{n-0,0}^{\dagger} \psi_{n-0}\right) \\
& +y \frac{1}{2} \sum_{n} \bar{\psi}_{n} \eta_{0}(n)\left(U_{n, 0} \psi_{n+0}+U_{n-0,0}^{\dagger} \psi_{n-0}\right)
\end{aligned}
$$

where $x$ and $y$ are two independent parameters. The QCD action is recovered from $S_{\tau}(x, y)$ by choosing $x=\cosh (\mu)$ and $y=\sinh (\mu)$.

Let us consider the generalized QCD action (2.3). A natural question is: what should we expect for the phase diagram of this model in the $x, y$ plane?

We are going to conjecture a minimal phase diagram for this generalized model of QCD. To this end let us analyze first the $y=0$ line. The point $x=1$ in this line would correspond to standard QCD at vanishing chemical potential. Now let be $L_{t}$ the temporal size of our lattice and assume we are in the scaling region but at a physical temperature $T$ lower than the deconfining critical temperature $T_{c}$. The point $x=1$ in the $y=0$ line will be in the confined phase. If we increase now the inverse gauge coupling $\beta$, the physical temperature increases and for $\beta$ values large enough the point $x=1$ will be in the unconfined phase. This strongly suggests the presence of a phase transition point in the $y=0$ line approaching the $x=1$ point in this line by increasing $\beta$ and eventually crossing it for $\beta$ values large enough. 


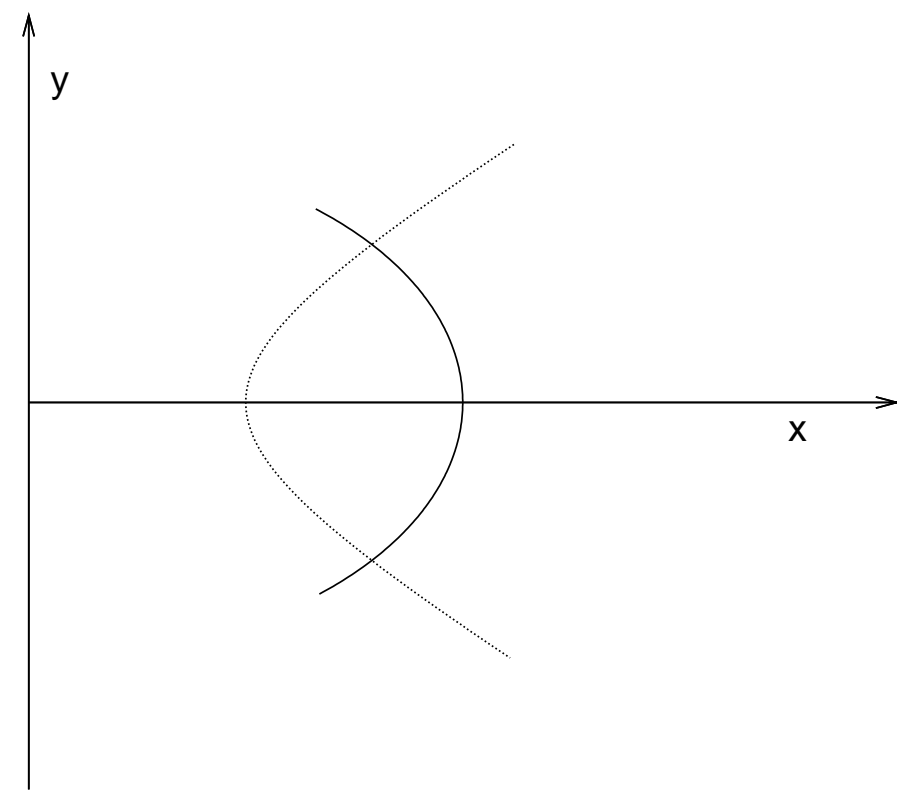

Figure 1: Minimal phase diagram conjectured for the generalized model of QCD. The solid line is a line of phase transitions. The discontinuous line is the physical line $x^{2}-y^{2}=1$.

This argument can be enforced if we consider the strong coupling limit. In this limit, where the pure gauge action vanishes, one can change the temperature by using symmetric lattices and changing the temporal coupling in the fermion action. The point $x=1$ in the $y=0$ line would be zero lattice temperature whereas $x>1$ would correspond to a temporal lattice spacing $a_{\tau}$ smaller than the lattice spacing $a$ in the space-like axis. Therefore $x>1$ corresponds, in the strong coupling limit, to finite lattice temperature. But even in the strong coupling limit one reaches a chiral phase transition by increasing the lattice temperature [10], thus suggesting that at $\beta=0$ there is a phase transition point in the $y=0$ line for a critical value of $x, x_{c}$, larger than 1 .

This argumentation drives one in a natural way to conjecture the minimal phase diagram shown in Fig. 1. The solid line would be a line of phase transitions that crosses the $y=0$ axis at values of $x$ larger than 1 for small $\beta$ values. By increasing $\beta$ and keeping fixed the temporal lattice extent $L_{t}$, the critical point on the $y=0$ axis moves toward $x=1$ and eventually crosses it. The discontinuous line in this figure stands for the physical line $x^{2}-y^{2}=1$ along which one recovers standard QCD. Along this line points with a coordinate $y \neq 0$ would correspond to non vanishing chemical potential whereas $x=1, y=0$ is $\mu=0$. The intersection of the solid line with the discontinuous one will therefore give us the critical chemical potential of $\mathrm{QCD}$ at a given temperature. A change in the physical temperature can be simulated by changing $\beta$ keeping fixed $L_{t}$ or vice-versa. In both cases the solid line in Fig.1 will move and the intersection point which gives the critical chemical potential will change with the physical temperature. The nature of the phase transition 
line will depend also on the other free parameters of the model i.e., number of flavors and quark masses.

\subsection{Symmetries of the partition function}

In order to get information on the symmetries of the partition function of the system described by the action (2.3), let us define the following change of variables in the path integral for the Grassmann fields,

$$
\psi_{j k}^{T}=e^{i k \alpha} \psi_{j k}, \quad \bar{\psi}_{j k}^{T}=e^{-i k \alpha} \bar{\psi}_{j k},
$$

where $k$ is the temporal coordinate of a given site, $j$ stands for the three spatial coordinates, $e^{i \alpha}$ is an element of the group $Z_{L_{t}}$ and $L_{t}$ is the number of time slices. The only piece of the action (2.3) not invariant under the previous variable change is $S_{\tau}(x, y)$, the different contributions of which transforming in the following way

$$
\begin{aligned}
\left(\bar{\psi}_{n} \eta_{0}(n) U_{n, 0} \psi_{n+0}\right)^{T} & =e^{\mathrm{i} \alpha}\left(\bar{\psi}_{n} \eta_{0}(n) U_{n, 0} \psi_{n+0}\right) \\
\left(\bar{\psi}_{n} \eta_{0}(n) U_{n-0,0}^{\dagger} \psi_{n-0}\right)^{T} & =e^{-\mathrm{i} \alpha}\left(\bar{\psi}_{n} \eta_{0}(n) U_{n-0,0}^{\dagger} \psi_{n-0}\right)
\end{aligned}
$$

In addition to the previous variable change for the Grassmann fields, we can also do a change of variables for the gluonic fields by multiplying each temporal link by an element $e^{i \gamma}$ of the center $Z_{3}$ of the $S U(3)$ group. Again the only non invariant contribution to (2.3) is $S_{\tau}(x, y)$ and its different pieces transform in the same way as before, replacing $e^{i \alpha}$ in (2.5) by $e^{i \gamma}$.

Using these transformations we can conclude that the integrated partition function of generalized QCD has the following symmetry

$$
Z_{V}(x+y, x-y)=Z_{V}\left(e^{\mathrm{i} \theta}(x+y), e^{-\mathrm{i} \theta}(x-y)\right),
$$

where $e^{\mathrm{i} \theta}$ is now an element of $Z_{3 L_{t}}$. Since the partition function must be independent of the parameters introduced by changing variables in the path integral, the only possibility is that it depends on $x, y$ through the combinations $(x+y)(x-y),(x+y)^{3 k L_{t}},(x-y)^{3 k L_{t}}$, with $\mathrm{k}$ any natural number. Taking also into account $C P T$ transformations we get that the dependence in the last two variables should appear as $(x+y)^{3 k L_{t}}+(x-y)^{3 k L_{t}}$, a result which could be also obtained by making the Polyakov loop expansion of the fermion determinant. Simple algebra tell us also that $(x+y)^{3 k L_{t}}+(x-y)^{3 k L_{t}}$ can be written as a combination of powers of $(x+y)^{3 L_{t}}+(x-y)^{3 L_{t}}$ and of $x^{2}-y^{2}$. Therefore we can say without loss of generality that the partition function $Z_{V}(x, y)$ will be, at finite volume, an analytic function $\bar{Z}_{V}(u, v)$ of the two variables

$$
\begin{aligned}
& u=x^{2}-y^{2}, \\
& v=(x+y)^{3 L_{t}}+(x-y)^{3 L_{t}},
\end{aligned}
$$

and this is a non trivial information that we are incorporating in the description of the model. 


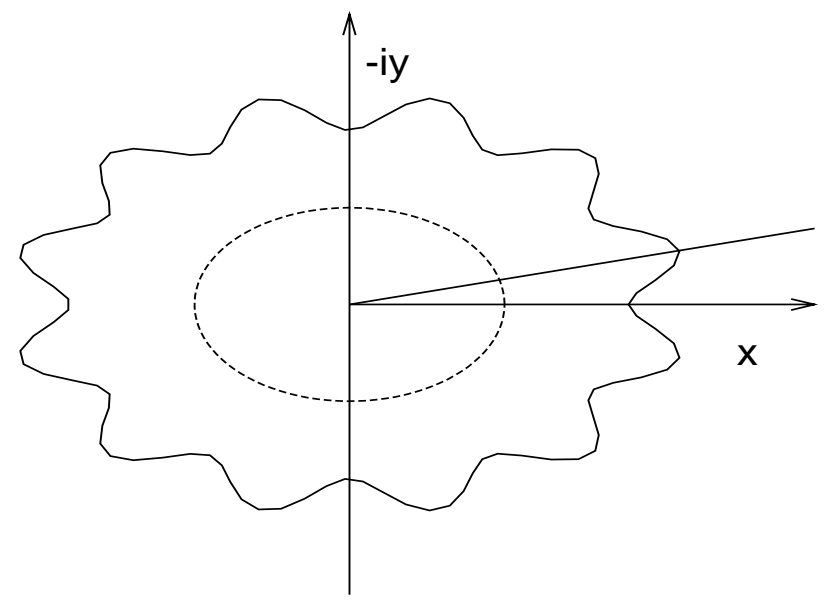

Figure 2: Conjectured phase diagram in the $x, \bar{y}$ plane. We have incorporated the property of periodicity. The dashed line contains the only points accessible to numerical simulations of $Q C D$ at imaginary chemical potential.

\subsection{The extension to imaginary y}

The numerical analysis of the model (2.3) for real values of $x, y$ is not possible since, as stated before, we find again the sign problem. However if we take the $y$ parameter as a pure imaginary number, $y=i \bar{y}, \bar{y}$ real, numerical simulations are feasible since the fermionic matrix, as for the case of imaginary chemical potential, is the sum of a constant diagonal matrix plus an antihermitian matrix, the last anticommuting with the $\gamma_{5}$ Dirac matrix. The eigenvalues of the fermion matrix appear then as pairs of complex conjugate numbers, and the fermion determinant is therefore real and positive.

Let be $z=x+i \bar{y}$ the complex number which we can associate to each pair of real values for $x, \bar{y}$. We can write $z$ as $z=\rho e^{i \eta}$ with

$$
\rho=\left(x^{2}+\bar{y}^{2}\right)^{1 / 2}, \quad \tan \eta=\frac{\bar{y}}{x} .
$$

The previous analysis on the symmetries of the partition function tell us that for imaginary values of the $y$ parameter, this function will depend only on $\rho$ and $\rho^{3 L_{t}} \cos \left(3 L_{t} \eta\right)$ i.e., the free energy will be a periodic function of $\eta$ with period equal to $2 \pi /\left(3 L_{t}\right)$. In particular if the phase transition line of Fig. 1 continues to imaginary values of $y$, the conjectured minimal phase diagram in the $x, \bar{y}$ plane would be that of Fig. 2, where we have incorporated the property of periodicity. 


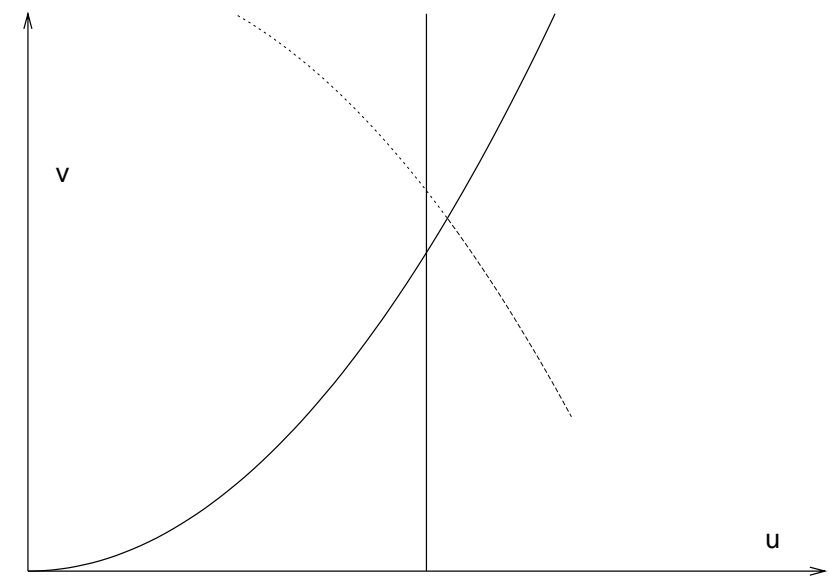

Figure 3: Phase diagram in the $(u, v)$ plane. The solid line $v=2 u^{\frac{3}{2} L_{t}}$ corresponds to the $y=0$ line of Fig. 1. The physical line $u=1$ and the hypothetical phase transition line are also included.

In Fig. 2 we have also included the line $\rho=1$ (dotted line) which contains the only points accessible to numerical simulations of $Q C D$ at imaginary chemical potential. As stated in the introduction, one can see now how this approach has in principle much more potentialities than the imaginary chemical potential approach. Indeed by increasing the inverse gauge coupling $\beta$, the phase transition line of Fig. 2 moves approaching more and more the origin of coordinates. In some interval $\left(\beta_{m}, \beta_{M}\right)$ the transition line intersects the $\rho=1$ line and then a phase transition will appear at imaginary chemical potential. In such a situation, the physical temperature is so high that the system is in an unconfined phase for any real value of the chemical potential. In order to understand something on the actual phase diagram from simulations at imaginary chemical potential, we need not only to extrapolate from imaginary to real values of $\mu$ but also to extrapolate from higher to lower temperatures or equivalently from higher to lower values of $\beta$. The advantage of our approach is that we can avoid the second kind of extrapolations because in our simulations $\rho$ is not enforced to be 1 .

This discussion can become more clever if we go from the $x, \bar{y}$ plane to the $u, v$ plane, where $u$ and $v$ are the variables defined in (2.7). The reason for choosing this plane is that it contains simultaneously the two planes of Figures 1 and 2. In Fig. 3 we have plotted the line

$$
v=2 u^{\frac{3}{2} L_{t}}
$$

which corresponds to the $y=0$ line of Fig. 1. We have also included in Fig. 3 the line $u=1$ which contains the discontinuous or physical line of Fig. 1 (points up the intersection point with the line $v=2 u^{\frac{3}{2} L_{t}}$ ) as well as the dotted line of Fig. 2 (points down the intersection point). All the plane of Fig. 2 maps into the region down the line $v=2 u^{\frac{3}{2} L_{t}}$ of Fig. 3, and this region is therefore accessible by simulating at imaginary $y$. On the other hand, 
the plane of Fig. 1 maps into the region up the solid line of Fig. 3. The only points in this Figure accessible to numerical simulations at imaginary chemical potential are those on the line $u=1$, down the intersection point with the line $v=2 u^{\frac{3}{2} L_{t}}$.

In Fig. 3 we include also a hypothetical but plausible realization of a phase transition line at a given physical temperature (simulations would be performed at fixed $\beta$ and $L_{t}$ ).

\subsection{Determination of the critical chemical potential near the continuum}

In order to get the critical value of the chemical potential we should determine the coordinates of the intersection point between the solid line and the physical line in Fig. 1. From the symmetries of the partition function we know that the phase transition line is an even function of $x$ and $y$. We can write therefore the following equation for the critical line of Fig. 1.

$$
x^{2}=1+a_{0}\left(\beta, L_{t}\right)+a_{2}\left(\beta, L_{t}\right) y^{2}+a_{4}\left(\beta, L_{t}\right) y^{4}+O\left(y^{6}\right),
$$

By fixing the lattice temporal extent $L_{t}$ and the gauge coupling $\beta$ one fixes the physical temperature $T$. The intersection point of the critical line with the physical line

$$
y_{c}^{2}=a_{0}\left(\beta, L_{t}\right)+a_{2}\left(\beta, L_{t}\right) y_{c}^{2}+a_{4}\left(\beta, L_{t}\right) y_{c}^{4}+O\left(y_{c}^{6}\right),
$$

will give us the critical value of the chemical potential $\left(y_{c}=\sinh \left(\mu_{c} a\right)\right)$ at this temperature.

If we approach the continuum limit by fixing the physical temperature $T$, i.e, by sending $\beta$ to $\infty$ keeping the product of $L_{t}$ times the lattice spacing $a$ fixed, we can write equation (2.11) in the following way

$$
\begin{aligned}
\left(\frac{\mu_{c}}{T_{c}}\right)^{2}+\frac{1}{3} \frac{\mu_{c}^{4}}{T_{c}^{2}} a^{2}+\cdots & =a_{0}\left(\beta, L_{t}\right) L_{t}^{2}+a_{2}\left(\beta, L_{t}\right)\left(\frac{\mu_{c}}{T_{c}}\right)^{2} \\
& +\left[a_{4}\left(\beta, L_{t}\right)+\frac{1}{3} a_{2}\left(\beta, L_{t}\right)\right] L_{t}^{-2}\left(\frac{\mu_{c}}{T_{c}}\right)^{4}+\ldots
\end{aligned}
$$

where in order to get this equation we have multiplied both sides of 2.11 by $L_{t}^{2}$. The coefficients $a_{k}\left(\beta, L_{t}\right)$ will depend in general on $L_{t}$ and on the dimensionless parameter $\Lambda / T$, where $\Lambda$ is the dimensional renormalization group parameter that fixes the QCD scale, and we have neglected for simplicity in all this analysis the dependence on the quark masses. Equation (2.12) then can be rewritten as follows

$$
\begin{aligned}
0 & =\bar{a}_{0}\left(\frac{\Lambda}{T}, L_{t}\right) L_{t}^{2}+\left(\bar{a}_{2}\left(\frac{\Lambda}{T}, L_{t}\right)-1\right)\left(\frac{\mu_{c}}{T_{c}}\right)^{2} \\
& +\left[\bar{a}_{4}\left(\frac{\Lambda}{T}, L_{t}\right)+\frac{1}{3} \bar{a}_{2}\left(\frac{\Lambda}{T}, L_{t}\right)-\frac{1}{3} a^{2} L_{t}^{2}\right] L_{t}^{-2}\left(\frac{\mu_{c}}{T_{c}}\right)^{4}+\ldots
\end{aligned}
$$

which suggests the following scaling laws for the coefficients

$$
\bar{a}_{k}\left(\frac{\Lambda}{T}, L_{t}\right)=c_{k}\left(\frac{\Lambda}{T}\right) \bar{a}_{0}\left(\frac{\Lambda}{T}, L_{t}\right) L_{t}^{k}
$$


The critical value of $\mu / T$ will be given by the solution of the equation (2.13). This equation suggests that for $\mu_{c} / T_{c}$ small, the determination of the first two coefficients of (2.10) could be enough for the computation of the critical chemical potential. The goodness of this approximation will depend on the values of the coefficients of (2.10). In the next section we show that this approximation works quite well in the Gross-Neveu model, even at large values of $\mu_{c} / T_{c}$.

The strategy for the determination of the critical chemical potential is then the following. From numerical simulations at imaginary values of $y, y=i \bar{y}$, near the phase transition point $\left(\left(1+a_{0}\right)^{1 / 2}, 0\right)$ one can locate several phase transition points in the $(x, \bar{y})$ plane (see Fig. 2). By fitting these points with equation (2.10) with the + sign of the coefficient proportional to $y^{2}$ replaced by -, we can numerically measure the first coefficients. The critical value of the chemical potential, $\mu_{c}$, will then be given by

$$
\mu_{c}= \pm \sinh ^{-1}\left(\frac{a_{0}}{1-a_{2}}\right)^{1 / 2}
$$

An alternative approach for the determination of the critical chemical potential is to use the variables $(u, v)$ instead of $(x, y)$. Since near the continuum $u$ takes values near 1 , the phase transition line of Fig. 3 could be fitted with the following equation

$$
v=b_{0}+b_{1}(u-1)+b_{2}(u-1)^{2}+O\left((u-1)^{3}\right) .
$$

The coefficient $b_{0}$ fully determines the critical $\mu$ through the following equation

$$
\mu_{c}=\frac{1}{3 L_{t}} \cosh ^{-1}\left(\frac{b_{0}}{2}\right)
$$

The use of the $(u, v)$ variables incorporates information on the symmetries of the free energy density. Furthermore consistency between results obtained from fits with equations (2.10) and (2.16) would improve the confidence level on the full procedure.

\subsection{Two other alternative ways for extracting the critical $\mu$ : the $(\beta, y)$ and $(\beta, x)$ planes}

In the previous paragraph it was assumed that the inverse gauge coupling $\beta$, the quark masses and the lattice temporal size $L_{t}$ were fixed and then we explored the phase diagram in the $(x, y)$ plane. In physical terms this means that, if $\beta$ is large enough to be in the scaling window, we fix the physical temperature $T$ and the physical quark masses. However it could be interesting in practice to analyze the projection of the phase diagram into the other two planes $(\beta, y)$ and $(\beta, x)$ for the following two reasons:

i. it would give an independent determination of $\left(\mu_{c}, T_{c}\right)$;

ii. one could apply some powerful techniques in the numerical analysis, as the FerrenbergSwendsen approach [11]. 


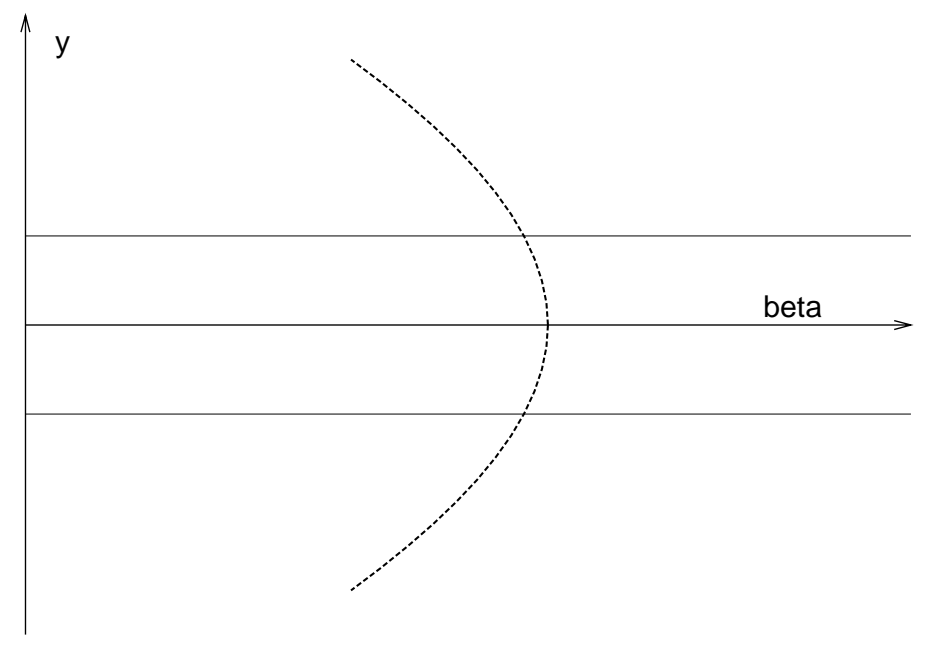

Figure 4: Conjectured phase diagram in the $(\beta, y)$ plane. The solid line is the physical line whereas the discontinuous one is a line of phase transitions.

Let us start with the case in which we fix $x=x_{0}>1, L_{t}$ and the lattice quark masses. The conjectured phase diagram in the $(\beta, y)$ plane is reported in Fig. 4. The solid line is the physical line

$$
y=y_{0}=\sinh \left(\cosh ^{-1}\left(x_{0}\right)\right),
$$

and the discontinuous line is a line of phase transitions. Using again the symmetries of the partition function we can write for this line the following equation

$$
\beta=\beta_{0}\left(x_{0}, L_{t}\right)+b_{2}\left(x_{0}, L_{t}\right) y^{2}+O\left(y^{4}\right),
$$

where we have neglected for simplicity the dependence of the coefficients on the quark masses.

We are interested in the determination of the intersection point between the physical and phase transition lines of Fig. 4. As in the case discussed in the previous section, one can measure $\beta_{0}$ and $b_{2}$ from simulations at $y=0$ and at imaginary $y=i \bar{y}$ (keeping $x_{0}$ fixed). Once we estimate the lattice spacing $a$, assumed we are in the scaling region, we can obtain $\beta_{c}, \mu_{c}$ and the critical values of the physical quark masses. Notice also that in the imaginary chemical potential approach $x_{0}$ is enforced to be less than 1 .

The last case to analyze, the projection of the phase diagram into the $(\beta, x)$ plane, is less interesting from a practical point of view because:

$i$. The physically relevant region in the $(\beta, x)$ plane (keeping $y=y_{0}$ fixed) is $x$ larger but near to 1 . The equation for the phase transition line near $x=1$ can therefore be written as

$$
\beta=\beta_{0}\left(y_{0}\right)+a_{1}\left(y_{0}\right)(x-1)+a_{2}\left(y_{0}\right)(x-1)^{2}+O\left((x-1)^{3}\right) .
$$


The critical inverse gauge coupling $\beta_{c}$ will be obtained by putting $x=x_{0}$ in (2.19), with

$$
x_{0}=\cosh \left(\sinh ^{-1}\left(y_{0}\right)\right) .
$$

But since no symmetry enforces now $a_{1}\left(y_{0}\right)$ to vanish, we should do, in contrast with the previous cases, a three parameters fit to get $\beta_{c}$ to order $\left(x_{0}-1\right)^{2}$.

ii. Numerical simulations must be performed at imaginary values of $y$ and therefore we need, at the end of the procedure, to do also an extrapolation of $\beta_{c}(y)$ from imaginary values of $y$ to real $y=y_{0}$.

\subsection{Vacuum expectation values on the phase transition line}

In order to get a deep understanding of the phase transition(s) at finite baryon density we would like to know not only the value of the critical chemical potential at a given physical temperature but also how vacuum expectation values of physical quantities change when crossing the phase transition line. To this end we will focus here on two typical observables, the mean plaquette energy and chiral condensate, and on the first theory scheme described previously i.e., the analysis of the phase diagram in the $x, y$ plane at fixed $\beta$. The generalization to other observables as the baryon number density, and, or to other projections of the three-dimensional phase diagram will be straightforward.

The chiral condensate $q\left(x^{2}, y^{2}\right)$ and plaquette energy $E\left(x^{2}, y^{2}\right)$ at fixed $\beta$ will be analytical even functions of $x$ and $y$ in all the points very near to the phase transition line both on the left and on the right of it. If we compute for instance the chiral condensate $q\left(x^{2}, y^{2}\right)$ in all the points of the phase transition line

$$
x^{2}=1+a_{0}\left(\beta, L_{t}\right)+a_{2}\left(\beta, L_{t}\right) y^{2}+a_{4}\left(\beta, L_{t}\right) y^{4}+O\left(y^{6}\right),
$$

approaching it from the left-hand side, we expect an analytical even function of $y$. Then we can write for the chiral condensate along this line the equation

$$
q_{c-}\left(y^{2}\right)=q_{c-}^{(0)}+q_{c-}^{(2)} y^{2}+O_{-}\left(y^{4}\right),
$$

and the same for all the points on the right of the phase transition line

$$
q_{c+}\left(y^{2}\right)=q_{c+}^{(0)}+q_{c+}^{(2)} y^{2}+O_{+}\left(y^{4}\right) .
$$

From simulations at imaginary $y=i \bar{y}$ one can measure the chiral condensate in both sides along the phase transition line, and by fitting the numerical results with these equations, we would get a numerical determination of the parameters in (2.20), (2.21). This procedure would allow us to extend the chiral condensate results to the physical point $y=y_{c}$.

\section{The Gross-Neveu model at finite density}

To check all the conjectures and ideas developed in section 2, we decided to use as a toy model the four-Fermi model in three dimensions with discrete chiral symmetry at finite $\mu$ and $T$. The continuum Lagrangian is 


$$
L=\sum_{j=1}^{N_{f}}\left[\bar{\psi}^{(j)} \not \partial \psi^{(j)}-\frac{g^{2}}{2 N_{f}}\left(\bar{\psi}^{(j)} \psi^{(j)}\right)^{2}\right],
$$

where $\psi^{(j)}$ is a four-component spinor, $N_{f}$ is the number of flavors and the discrete $Z_{2}$ symmetry is $\psi \rightarrow \gamma_{5} \psi, \bar{\psi} \rightarrow-\bar{\psi} \gamma_{5}$. This model has a non trivial renormalization group fixed point characterized by the spontaneous breaking of the chiral symmetry at a finite critical coupling $g_{c}^{2}$, its $1 / N_{f}$ expansion about the fixed point $g_{c}^{2}$ being renormalizable. In addition the model can be solved analytically in the large $N_{f}$ limit and then we can compare numerical predictions with exact results in this limit. Finally the phase structure of the model in the $(\mu, T)$ plane resembles to that expected for QCD, with a phase transition line separating a phase where chiral symmetry is spontaneously broken from a phase where the symmetry is restored.

After the introduction of an auxiliary scalar field $\sigma$, equation (3.1) becomes

$$
L=\sum_{j=1}^{N_{f}}\left[\bar{\psi}^{(j)} \not \partial \psi^{(j)}+\sigma \bar{\psi}^{(j)} \psi^{(j)}\right]+\frac{N_{f}}{2 g^{2}} \sigma^{2},
$$

the vacuum expectation value of $\sigma$ being a good order parameter for the chiral symmetry restoration transition.

Regularized in a space-time lattice with staggered fermions, the Gross-Neveu action is 9

$$
\begin{aligned}
S & =\sum_{i=1}^{N_{f} / 2} \sum_{n} \sum_{\mu=1}^{3} \frac{1}{2} \eta_{\mu}(n) \bar{\psi}_{i}(n)\left[\psi_{i}(n+\mu)-\psi_{i}(n-\mu)\right] \\
& +\frac{1}{8} \sum_{i=1}^{N_{f} / 2} \sum_{n} \bar{\psi}_{i}(n) \psi_{i}(n) \sum_{<\bar{n}, n>} \sigma(\bar{n})+\frac{N_{f}}{4 g^{2}} \sum_{\bar{n}} \sigma^{2}(\bar{n}),
\end{aligned}
$$

where the symbol $\langle\bar{n}, n>$ denotes the set of eight dual lattice sites $\bar{n}$ surrounding the lattice site $n, \eta_{0}(n)=1$ and $\eta_{\mu}(n)=(-1)^{n_{\mu}+n_{\mu-1}}$ for $\mu=1,2$.

The inclusion of a chemical potential $\mu$ selecting a finite fermion density is achieved, as in QCD, by multiplying all forward (backward) temporal links by $e^{\mu},\left(e^{-\mu}\right)$. The fermionic bilinear part of the action then becomes

$$
\begin{aligned}
S_{F} & =\sum_{i=1}^{N_{f} / 2} \sum_{n} \frac{1}{2} \bar{\psi}_{i}(n)\left[e^{\mu} \psi_{i}(n+0)-e^{-\mu} \psi_{i}(n-0)\right] \\
& +\sum_{i=1}^{N_{f} / 2} \sum_{n} \sum_{\alpha=1}^{2} \frac{1}{2} \bar{\psi}_{i}(n) \eta_{\alpha}(n)\left[\psi_{i}(n+\alpha)-\psi_{i}(n-\alpha)\right] \\
& +\frac{1}{8} \sum_{i=1}^{N_{f} / 2} \sum_{n} \bar{\psi}_{i}(n) \psi_{i}(n) \sum_{<\bar{n}, n>} \sigma(\bar{n})
\end{aligned}
$$



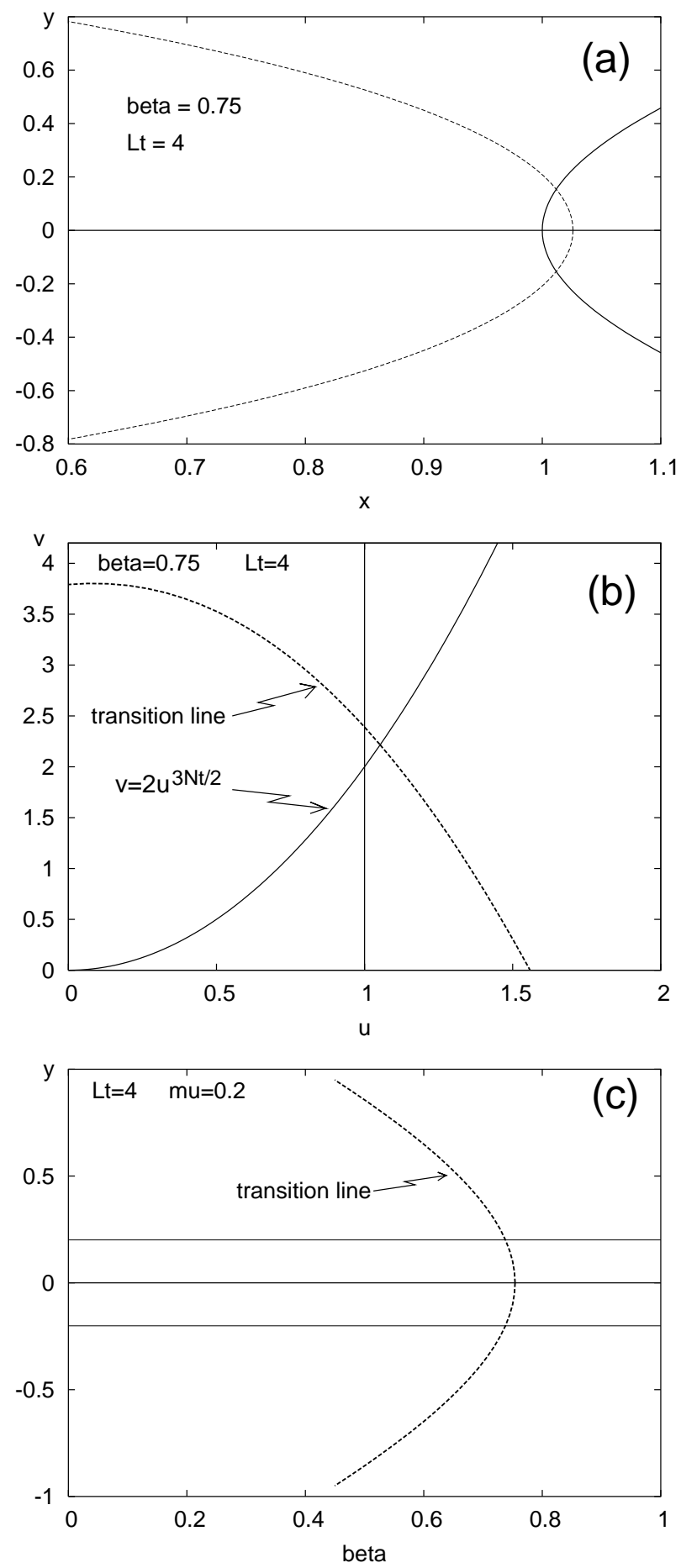

Figure 5: Phase diagrams of the generalized four-Fermi model in the $(x, y)(5 \mathrm{a}),(u, v)(5 \mathrm{~b})$ and $\left(1 / g^{2}, y\right)(5 \mathrm{c})$ planes respectively. All the phase diagrams correspond to a lattice temperature $1 / L_{t}=1 / 4$ in lattice units. The inverse four-Fermi coupling $1 / g^{2}=\beta=0.75$ in Figs. 5a and 5b whereas $x=1.0211$ in Fig. 5c. The solid line is the physical line whereas the discontinuous line is a second order phase transition line. 
In the large number of flavors limit, $N_{f} \rightarrow \infty$, fluctuations around the saddle point solution are suppressed and then the vacuum expectation value $\Sigma$ of the scalar field $\sigma$ can be self-consistently determined using the gap equation

$$
\Sigma=-g^{2}\langle\bar{\psi} \psi\rangle
$$

where $\langle\bar{\psi} \psi\rangle$ here means the mean value of the chiral condensate in the free fermion theory at finite chemical potential and with an effective fermion mass $m=\Sigma$.

The formalism developed in the previous section can be straightforwardly applied here. Indeed we can define a generalized fermion action

$$
\begin{aligned}
S_{F}(x, y) & =x \frac{1}{2} \sum_{i=1}^{N_{f} / 2} \sum_{n} \bar{\psi}_{i}(n)\left[\psi_{i}(n+0)-\psi_{i}(n-0)\right] \\
& +y \frac{1}{2} \sum_{i=1}^{N_{f} / 2} \sum_{n} \bar{\psi}_{i}(n)\left[\psi_{i}(n+0)+\psi_{i}(n-0)\right] \\
& +\sum_{i=1}^{N_{f} / 2} \sum_{n} \sum_{\alpha=1}^{2} \frac{1}{2} \bar{\psi}_{i}(n) \eta_{\alpha}(n)\left[\psi_{i}(n+\alpha)-\psi_{i}(n-\alpha)\right] \\
& +\frac{1}{8} \sum_{i=1}^{N_{f} / 2} \sum_{n} \bar{\psi}_{i}(n) \psi_{i}(n) \sum_{<\bar{n}, n>} \sigma(\bar{n}),
\end{aligned}
$$

that agrees with action (3.4) for $x=\cosh (\mu), y=\sinh (\mu)$. In the large $N_{f}$ limit the generalized model can also be analytically solved, and the solution is again given by the gap equation (3.5) where now $\langle\bar{\psi} \psi\rangle$ means the mean value of the chiral condensate in the generalized free-fermion model at an effective fermion mass $m=\Sigma$.

We are now in good conditions to check if the conjectures formulated in section 2 are realized in the four-fermion model. Figures $5 \mathrm{a}, 5 \mathrm{~b}$ and $5 \mathrm{c}$ show the phase diagrams of the generalized four-Fermi model in the $(x, y),(u, v)$ and $\left(y, g^{2}\right)$ planes respectively. All the phase diagrams correspond to a lattice temperature $1 / L_{t}=1 / 4$ in lattice units. The inverse four-Fermi coupling $\beta=1 / g^{2}=0.75$ in Figs. 5a and 5b whereas $x=1.0211(\mu=0.2)$ in Fig. 5c. A second order phase transition line separating a broken from an unbroken phase and crossing the physical line $x=\cosh \mu, y=\sinh \mu$ was found in all the cases, thus confirming actually all our conjectures.

To have a guess on the potentialities of our approach when applied to QCD we have done a further check. This check consisted in fitting several phase transition points in the $(x, y)$ plane at imaginary $y$ with equation (2.10) in the quadratic approximation (twoparameter fits). The purpose of the check was to assume these phase transition points as they were obtained from numerical simulations at imaginary $y$, where the sign problem is absent, and then to check the degree of precision with which we can predict $\mu_{c}$ and $T_{c}$.

To locate the physical region in which the agreement between the extrapolated results and the exact ones is better, we have plotted in Fig. 6 the phase diagram in the $\left(\mu / T, T / \Sigma_{0}\right)$ plane for several values of the lattice temporal extent $L_{t} . \Sigma_{0}$ is the expectation value of 


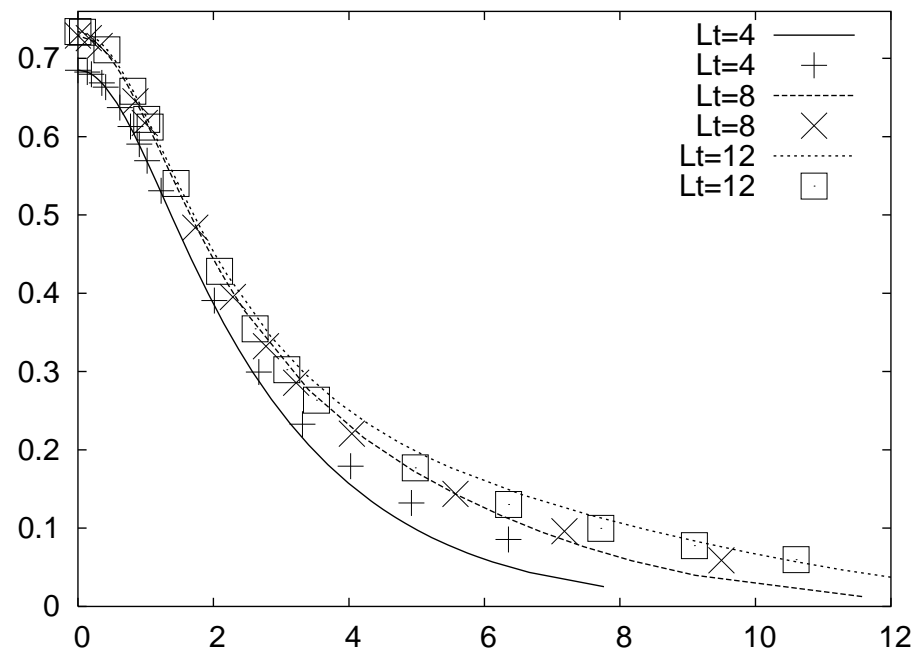

Figure 6: Phase diagram in the $\left(\mu / T, T / \Sigma_{0}\right)$ plane for several values of the lattice temporal extent $L_{t} . \quad \Sigma_{0}$ is the expectation value of the scalar field $\sigma$ in the ground state at $T=0$. The lines correspond to analytical results at the same values of $L_{t}$ while the symbols correspond to the extrapolated results.

the scalar field $\sigma$ in the ground state at $T=0$, and the plots have been done assuming asymptotic scaling. The lines correspond to analytical results. The observed dependence on $L_{t}$, especially at large $\mu / T$, shows that the effects of regularization are still important in this region for these lattices. One can also observe in this figure how the extrapolated results agree very well with the exact ones even at large values of $\mu / T$.

Finally and in order to confront our method with the imaginary chemical potential approach, we have plotted in Fig. 7 the phase diagram of the Gross-Neveu model in the $\mu, 1 / g^{2}$ plane as obtained from the analysis of the $x, y$ plane at imaginary $y$ in the quadratic approximation of equation (2.10) (crosses), and from the imaginary chemical potential approach (dashed lines), the last obtained from quadratic as well as quartic fits of the critical coupling as a function of the imaginary chemical potential. The solid line stands for the exact results on a lattice of temporal extent $L_{t}=4$. As can be seen both approaches agree with the analytical results in the small chemical potential region, but results obtained with our method follow quite well the exact ones in the large $\mu$ region in contrast with the imaginary chemical potential results that give even negative values for $g_{c}^{2}$ at large $\mu$ in the quadratic approximation. From both theoretical and practical points of view there is little hope to get reliable values for more than two-three coefficients from imaginary $\mu$ numerical data, so we improved the fit procedure, as stated before, considering up to quartic fits of the critical coupling as a function of the imaginary chemical potential. As can be seen in Fig. 7 the extra degree of freedom improves the results at small $\mu$, but not in a relevant way. 


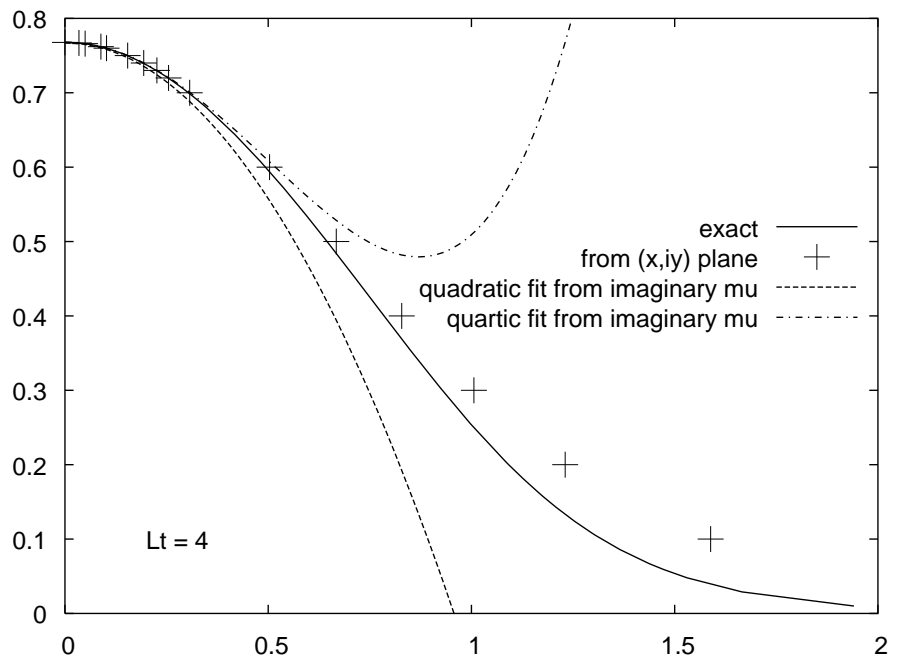

Figure 7: Phase diagram of the Gross-Neveu model in the $\mu, 1 / g^{2}$ plane as obtained from the analysis of the $x, y$ plane at imaginary $y$ (crosses), and from the imaginary chemical potential approach (dashed line). The solid line stands for the exact result on a lattice of temporal extent $L_{t}=4$

\section{Four flavor QCD at finite $\mathrm{T}$ and $\mu$}

Understanding the finite temperature and finite density phase structure of QCD is one of the main challenges of lattice field theory. Whereas qualitative and quantitative progress on the phase structure of the model at vanishing baryon density has been achieved in the last years, the sign problem has prevented, as discussed in the introduction of this paper, any significant progress on the understanding of the behavior of matter at high density.

We want to devote this section to the application of the approach described in the previous sections to the analysis of the phase diagram of four flavor QCD at finite baryon density. The aim of such an analysis is twofold: first we want to verify if the conjectures formulated in the first section, and corroborated in the previous section for the Gross-Neveu model, do actually work in QCD, and second we want to gain some experience in order to analyze in a future work the more interesting cases of 2 or $2+1$ flavor QCD.

Even if a good understanding of the phase diagram of the four flavor model would be of interest, the aim of this section is not to do an exhaustive analysis of the four flavor model but rather to verify if the approach reported in this paper can hopefully be applied to QCD. This is the reason why we have concentrated our efforts on the analysis of one point of the phase diagram, but using two of the independent approaches described in section 2: the determination of the critical values of $\mu$ and $T$ from the analysis of the phase diagrams in the $(x, y)$ and $(\beta, y)$ planes. Since the two approaches are based on independent numerical simulations and independent fits, the agreement between the predicted critical parameters should be considered a proof of the reliability of the results. 


\subsection{The $(x, y)$ plane}

The model analyzed is therefore that described by action (2.1) i.e., QCD at finite $\mu$ and four degenerate staggered flavors. In order to determine a point of the phase transition line in the $(T, \mu)$ plane we performed HMC simulations of the generalized model described by action (2.3), (2.4) at several values of $x$ and imaginary values of $y=i \bar{y}$. The lattice size was $8^{3} \times 4$, the inverse gauge coupling $\beta=5.05$, and the quark masses were fixed to $m a=0.06$ in lattice units. The corresponding physical temperature is $T \approx 136 \mathrm{MeV}$.

We have performed simulations for 7 values of $\bar{y}$ from 0 to 0.15 ; for each value of $y$ $8-13$ values of $x$ have been simulated with statistics ranging from 10000 to 50000 HMC trajectories (the larger ensembles near the critical points). The simulations have been performed on the Linux clusters of LNGS-INFN, using a total of 50 CPUs.

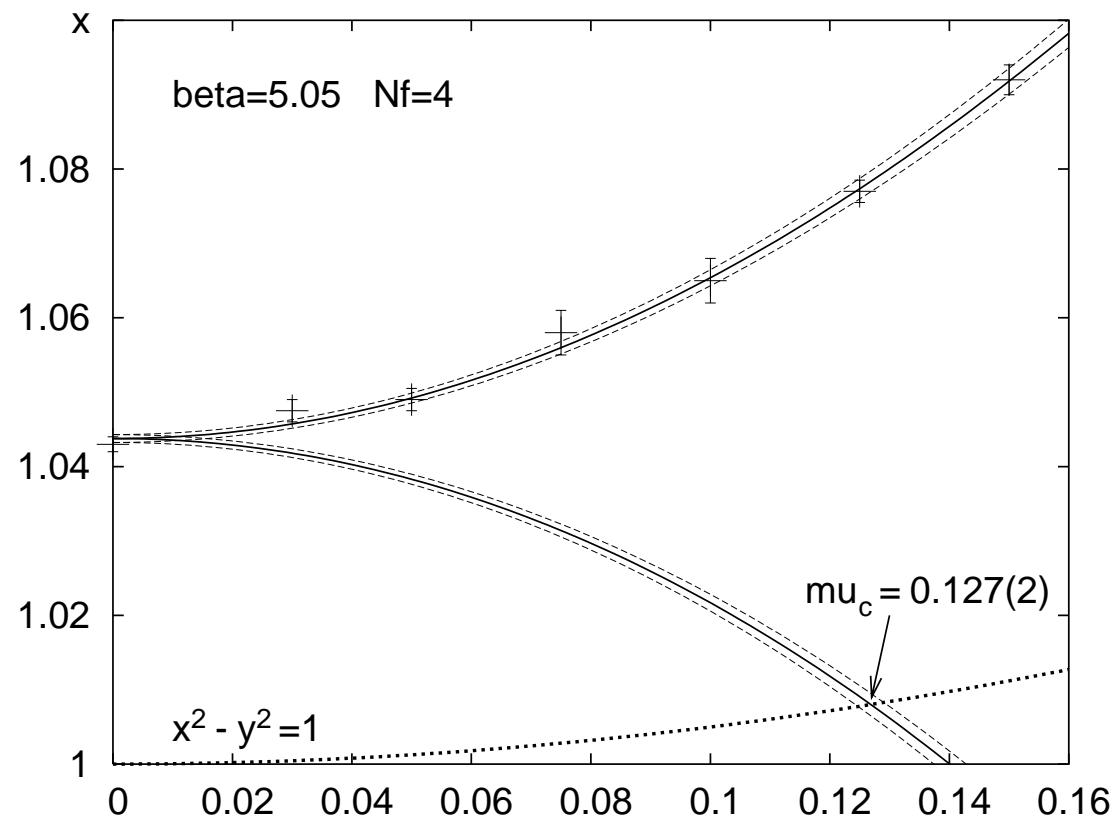

Figure 8: Measured critical points in the $(\bar{y}, x)$ plane at $\beta=5.05$ and $m a=0.06$. The upper and lower half figure correspond to imaginary and real $y$ respectively. The solid lines are a quadratic fit of the points and its analytical continuation to real $y$. We include also the physical line $x^{2}-y^{2}=1$. Dashed lines represent maximum deviation of the fitting function within statistical errors.

From these numerical simulations we have determined seven critical points in the $(\bar{y}, x)$ plane (see Fig. 8), looking for the position of the maximum of the plaquette, chiral, and Polyakov loop susceptibilities. The position of the maximum was compatible for the three observables in all the cases, but the results for the plaquette susceptibility seem to be of better quality. The two-state signal observed for the three measured quantities in all the points plotted in Fig. 8 indicates a first order phase transition line. Unfortunately we have no data on larger lattices which would allow a finite size scaling analysis to confirm these expectations. 
By fitting the seven points of Fig. 8 with a quadratic function (equation (2.10) with $a_{n}=0$ for $n>2$ ) we get the solid line in this Figure. Its analytical continuation to real values of $y$ has also been plotted in Fig. 8 where one can also observe the intersection point with the physical line $x^{2}-y^{2}=1$. The coordinates of this intersection point $x_{c}=$ $1.0081(2), y_{c}=0.127(2)$ allow us to predict that the critical chemical potential in lattice units is $\mu_{c}=0.127(2)$ at $\beta=5.05$ and $m a=0.06$.

We can fix the lattice scale from the mass of the $\rho$ resonance, $770 \mathrm{MeV}$. In such case the results of reference [12] imply that the lattice spacing is approximately $0.3 \mathrm{fm}$ at $\beta=5.2$ for $m a=0.06$, and we set the lattice spacing at smaller values of $\beta$ by using the two loop $\beta$ function. The phase transition at $\mu=0$ is located at $\beta \approx 5.08$ [13]. This gives $T_{\mathrm{C}} \approx 136 \mathrm{MeV}$. Our results reported in the previous paragraph give another point of the phase transition line: $T_{\mathrm{C}} \approx 133 \mathrm{MeV}, \mu_{\mathrm{C}} \approx 68(1) \mathrm{MeV}$.

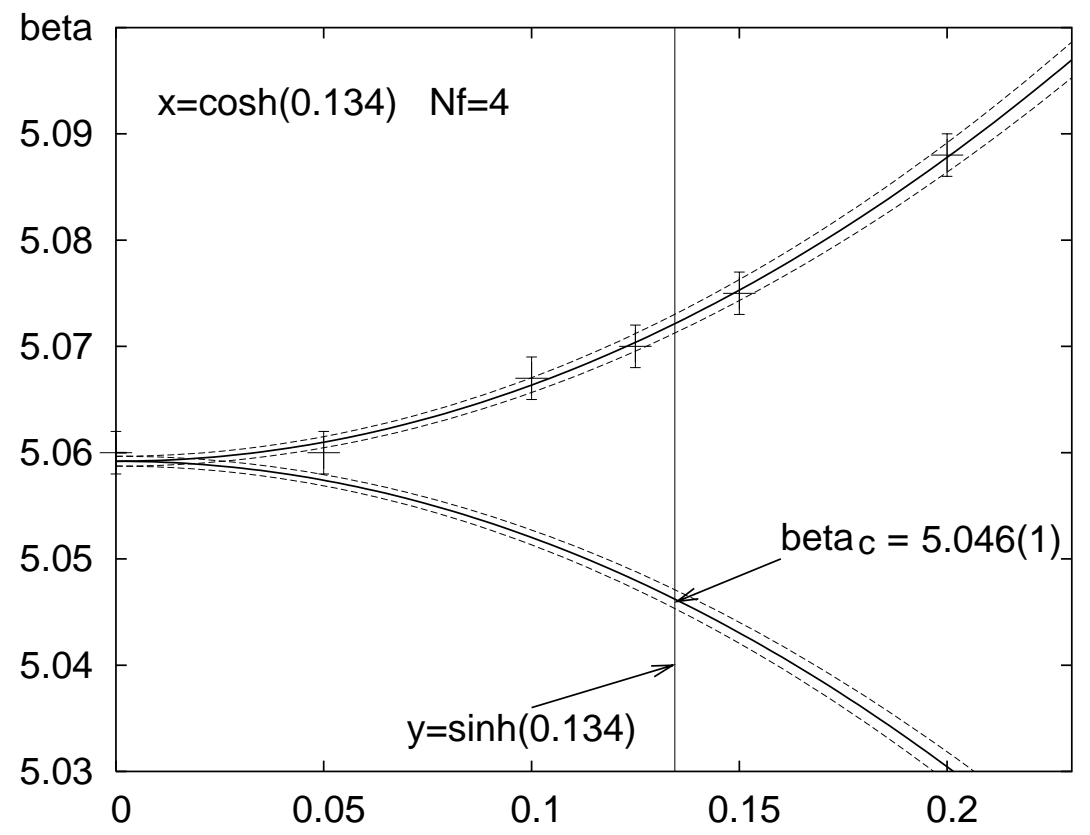

Figure 9: Measured critical points in the $(\bar{y}, \beta)$ plane at $x=1.009$ and $m a=0.06$. The upper and lower half figure correspond to imaginary and real $y$ respectively. The solid lines are a quadratic fit of the points and its analytical continuation to real $y$. We include also the physical line $y=$ $\sinh \left(\cosh ^{-1}(1.009)\right)$. Dashed lines represent maximum deviation of the fitting function within statistical errors.

Once we have determined the coordinates of the critical point, we can apply the formalism described in section 2.6 to analyze the nature of the physical phase transition. A very interesting feature that emerges from our simulations is that the latent heat is independent of the parameters $x, y$ within errors in such a way that there is not a real need to perform an analytical continuation; therefore the physical phase transition is also first order. Similar results have also been obtained for the chiral condensate and Polyakov loop. 


\subsection{The $(\beta, y)$ plane}

By fixing $x=1.009$ (corresponding to $\mu=0.134$, our first rough estimation of the critical value at $\beta=5.05$ and $m a=0.06)$, we have also performed simulations in the $(\beta, \bar{y})$ plane, using statistics and number of values for the parameters $\beta$ and $\bar{y}$ similar to those of the $(x, y)$ case. The aim of this calculation, as stated before, is to check if we get results consistent with those of section 4.1 .

Fig. 9 is the same as Fig. 8 but in the $(y, \beta)$ instead of the $(y, x)$ plane. The solid lines are a fit of the measured phase transition points with equation (2.18) and its analytical continuation to real values of $y$. Again in this case a clear two state signal was observed for the three measured observables. As in Fig. 8, we have also plotted in Fig. 9 the physical line that in this case is the line $y=\sinh \left(\cosh ^{-1}(1.009)\right)$. The intersection point of the phase transition line with the physical line gives the critical value $\beta_{c}=5.046(1)$ in close agreement with the results reported in the previous section, if we take in mind that the value of $\mu$ used (0.134) is slightly larger than the actual critical $\mu$ at $\beta=5.05$ (0.127). For this case the critical point is $T_{\mathrm{C}} \approx 132 \mathrm{MeV}, \mu_{\mathrm{C}} \approx 71 \mathrm{MeV}$. Also for this set of simulations the global behavior of latent heat suggests a first order transition.

\section{Conclusions and comments}

We have introduced a new approach to analyze the phase diagram of QCD at finite chemical potential and temperature, and applied it to the four-fermion model in three dimensions and to QCD with four degenerate quarks.

Our scheme is based on the introduction of a generalized QCD action which depends on two extra free parameters, $x, y$. When the point $(x, y)$ belongs to the curve $x^{2}-y^{2}=1$ one recovers standard QCD at finite chemical potential.

The generalized QCD action suffers also, for real values of $y$, from the sign problem. However at imaginary values of $y$ the fermion determinant is positive definite and therefore standard numerical simulations are feasible.

Even if our theory scheme can resemble the imaginary chemical potential approach, we have shown that its range of applicability is much wider. Indeed our approach shares with the imaginary chemical potential method the need of analytical. However whereas imaginary chemical potential simulations have to be performed at high temperature (large $\beta$ ) i.e., in the deconfined phase for real values of $\mu$, in our scheme simulations can be performed at any temperature and any value of $\mu / T$. The price to pay for that is one more parameter in the numerical procedure.

To make evident the potentialities of our approach we have confronted it with the imaginary chemical potential method when applied to the Gross-Neveu model in the limit of large number of flavors, where analytical results are available. We have shown how both approaches agree with the analytical results in the small chemical potential region, but results obtained with our method follow quite well the exact ones in the large $\mu$ region in contrast with the imaginary chemical potential results, that give even negative values for

$g_{c}^{2}$ at large $\mu$. An open point however is to dispose of a good criterion to understand in which region of the parameters space the quadratic approximation of equation (2.10) (or 
equivalently of equation (2.13) ) works well. For what concerns the Gross-Neveu model, the aforementioned region is actually very wide.

We have also performed simulations of QCD with four degenerate flavors in order to test the procedure in a real QCD simulation. We have determined the critical chemical potential for a value of $\beta$ inside the scaling region and, using the second scheme depicted in section 2.5 , we found the critical $\beta$ for a given $\mu$ value, obtaining a consistent pair of results.

As stated before, our theory scheme requires more computer time than other more standard simulations since we have one more parameter in the action. Roughly speaking the computer time needed to get a single point of the phase diagram in the $(\mu, T)$ plane is of the order of the one needed to get the critical line within the imaginary chemical potential approach. However, our approach, as we have shown for the Gross-Neveu model, seems able to reproduce quite well the phase diagram at values of $\mu / T$ not accesible to the other standard methods, and this is in our opinion the reason that makes it relevant for the field despite of its computing cost.

Concerning the Glasgow reweighting procedure in two parameters space [7], one could consider the possibility of using the fermion determinant at the critical point of the $y=0$ line instead of the determinant at $\mu=0$ in the integration reweighted measure. We suspect that hereby one could improve the overlaps.

Finally we plan for near future to apply our approach to the analysis of two-color QCD at finite $\mu$ and to 2 and $2+1$ flavor QCD.

\section{Acknowledgments}

This work has been partially supported by an INFN-CICyT collaboration and by Ministerio de Ciencia y Tecnología (Spain), projects FPA2003-02948 and BFM2003-08532-C0301/FISI. V.L. has been supported by Ministerio de Ciencia y Tecnología (Spain) under the Ramón y Cajal program. 


\section{References}

[1] I.M. Barbour, S.E. Morrison, E.G. Klepfish, J.B. Kogut, M.P. Lombardo, Phys. Rev. D 56 (1997) 7063.

[2] R. Aloisio, V. Azcoiti, G. Di Carlo, A. Galante, A.F. Grillo, Phys. Lett. B 428 (1998) 166; Phys. Lett. B 435 (1998) 175.

[3] V. Azcoiti, G. Di Carlo, A.F. Grillo, Phys. Rev. Lett. 65 (1990) 2239.

[4] R. Aloisio, V. Azcoiti, G. Di Carlo, A. Galante, A.F. Grillo; Phys. Rev. D 61 (2000) 111501R.

[5] P. de Forcrand, O. Philipsen, Nucl. Phys. B 642 (2002) 290; Nucl. Phys. B 673 (2003) 170.

[6] M. D'Elia, M.P. Lombardo, Phys. Rev. D 67 (2003) 014505. M.P Lombardo, Prog. Theor. Phys. Suppl. 153 (2004) 26.

[7] Z. Fodor, S.D. Katz, Phys. Lett. B 534 (2002) 87; J. High Energy Phys. 0203 (2002) 014; J. High Energy Phys. 0404 (2004) 050.

[8] C. R. Allton, S. Ejiri, S. J. Hands, O. Kaczmarek, F. Karsch, E. Laermann, Ch. Schmidt, L. Scorzato, Phys. Rev. D 66 (2002) 074507. S. Ejiri, C. R. Allton, S. J. Hands, O. Kaczmarek, F. Karsch, E. Laermann, C. Schmidt, Prog. Theor. Phys. Suppl. 153 (2004) 118.

[9] S. Hands, A. Kocic, J.B. Kogut, Nucl. Phys. B 390 (1993) 355.

[10] A. Moreo, Phys. Rev. D 35 (1987) 1442.

[11] A.M. Ferrenberg, R.H. Swendsen, Phys. Rev. Lett. 61 (1988) 2635.

[12] K.D. Born, E. Laerman, N. Pirch, T.F. Walsh, P.M. Zerwas, Phys. Rev. D 40 (1989) 1653.

[13] A. Hasenfratz, F. Knechtli, hep-lat/0105022. 